\title{
Recaídas en la adicción a cocaína: una revisión
}

\section{Relapse in cocaine addiction: a review}

\author{
Emilio SánChez-Hervás*; \\ JuAn Miguel Llorente del Pozo **
}

* Unidad de Conductas Adictivas de Catarroja. Agencia Valenciana de Salud
${ }^{* \star}$ Hospital de Día de Adicciones - Osakidetza. Vitoria

\section{Resumen}

Las recaídas siguen siendo un problema común en los trastornos relacionados con el consumo de cocaina. El objetivo de este trabajo es el de revisar los últimos datos de la investigación sobre las variables y factores relacionados con las recaídas en el consumo en personas dependientes a la cocaina. Se realizó una búsqueda electrónica de trabajos que incluyeran el estudio de variables relacionadas con la recaida en el consumo de cocaina, en algunas de las bases de datos nacionales e internacionales más importantes en la actualidad: PubMed, ISI Web of Knowledge, The Cochrane Library, IME e ISOC. Para localizar los últimos datos de investigación la búsqueda se realizó para estudios publicados en el periodo comprendido entre Enero de 2000 a Julio de 2011. Han sido varios y variados los factores y variables identificadas con cierto poder predictivo, entre los que destacan la problemática psicosocial, los estados emocionales negativos, la ansiedad, la autoeficacia, la comorbilidad psicopatológica, la severidad adictiva, y varias variables relacionadas con el proceso de tratamiento. Los conocimientos sobre los procesos de recaida se han incrementado considerablemente en los últimos años, y aunque la influencia de algunos factores sobre las recaídas en el consumo de cocaina parece clara, la investigación especifica es todavia escasa y no concluyente.

Palabras clave: cocaina, recaídas, revisión, resultados de tratamiento.

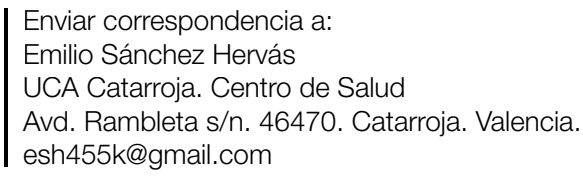


S egún los últimos datos epidemiológicos disponibles se estima que 14 millones de europeos consumieron cocaína alguna vez en la vida, que 4 millones lo hicieron en el último año, y que 2 millones lo hicieron en el último mes (Observatorio Europeo de las Drogas y las Toxicomanías, 2010). En España el número de personas tratadas como consecuencia del uso de esta sustancia ha pasado de poco más de 900 en el año 1991 a más de 22.000 en el 2007 (Observatorio Español sobre Drogas, 2009). Además, la prevalencia de consumo en los últimos años se situó en torno al $3 \%$, una de las cifras más altas de la Unión Europea (Oficina de las Naciones Unidas contra la Droga y el Delito, 2009). La tendencia en el consumo de cocaína en Europa ha seguido patrones diferentes; en los dos países con mayor prevalencia (España y el Reino Unido) el consumo aumentó de forma espectacular en la década de los noventa, para pasar a una tendencia más estable, pero por lo general al alza (Observatorio Europeo de las Toxicomanías, 2010).

Aunque se están realizando ensayos con algunos fármacos prometedores, en la actualidad ningún tratamiento farmacológico ha sido considerado eficaz para tratar la dependencia a la cocaína (Montoya y Vocci, 2008). Por otra parte algunos programas de tratamiento psicológico han encontrado resultados positivos en el tratamiento de esta adicción (Dutra et al., 2008; Knapp, Soares, Farrell y Silva de Lima, 2007; Ochoa, Madoz-Gurpide y Caballero, 2011; Shearer, 2007).

Las posibles alternativas terapéuticas a esta problemática presentan a día de hoy algunas lagunas. A pesar de algunos avances conseguidos en los últimos años, las recaídas en el consumo y los períodos de uso incontrolado siguen siendo un problema común; la mayoria de las personas que buscan tratamiento no son capaces de mantener un período de abstinencia continuada (Witkiewitz y Marlatt, 2004). La mayor parte de ellas no logran realizar cambios efectivos en su primer intento de tratamiento, las recaídas son frecuentes y las tasas de cumplimiento de los programas ofrecen unos resultados moderados (Dutra et al., 2008). Esta falta de adherencia a los tratamientos y las frecuentes recaídas enturbian el pronóstico de estos pacientes.

El retorno de un paciente al uso de cocaína después de un período más o menos prolongado de abstinencia, puede ser visto como un evento temporal o como una vuelta al uso persistente. En realidad, existen dificultades para establecer un estándar formal que distinga el uso de cocaína (y de drogas en general) de forma persistente, de una recaída ocasional. Independientemente de cómo se defina una recaída, la investigación ha demostrado que la mayoría de las personas que tratan de cambiar su comportamiento en una determinada dirección (por ejemplo, perder peso, reducir la hipertensión arterial, dejar de fumar, etc) experimentarán lapsos (caidas o errores) que a menudo conducirán a una recaída (Polivy y Herman, 2002).

En general las tasas de recaída en adicciones son altas, especialmente en la adicción a cocaína, y quizás constituyen el problema más difícil al que enfrentan los investigadores y los clínicos que trabajan en el ámbito de la las conductas adictivas. La mayoria de los profesionales reconocen que si bien abandonar un comportamiento problemático como el uso de cocaína puede ser difícil, mantener un estilo de vida sin retornar a dicho comportamiento puede llegar a ser aún más complicado. Para los autores de la revisión una recaída se puede definir de forma adecuada según la versión clásica del modelo de prevención de recaídas (Marlatt y Gordon, 1985); como un proceso transitorio que puede o no estar seguido por el regreso a los niveles de línea base de la conducta objetiva observable antes del tratamiento.

\section{Factores predictivos de recaída}

Basándose en el modelo clásico de prevención de recaídas de Marlatt (Marlatt y Gordon, 1985), en los años ochenta se identificaron tres situaciones de alto riesgo principales que se asociaban con casi las tres cuartas partes de todas las recaídas: estados emocionales, conflictos interpersonales y presión social. Estas tres situaciones de riesgo se consideraban frecuentemente asociadas a la recaída, independientemente del problema de que se tratara (Cummings, Gordon y Marlatt, 1980). En los últimos años, el modelo clásico de prevención de recaídas se ha reconceptualizado y ha ampliado la naturaleza de las relaciones entre los procesos implicados en la recaída (Witkiewitz y Marlatt, 2004). En esta nueva perspectiva se aporta una comprensión multidimensional, dinámica y sistémica, para explicar la compleja interacción de los factores que intervienen en ellos. En el modelo original se ofrecia una interpretación lineal (estática) del proceso, mientras que en este nuevo planteamiento se asume la existencia de influencias reciprocas entre variables disposicionales, contextuales y las experiencias pasadas y actuales de los pacientes. Se plantea la importancia de diversos factores para desencadenar y actuar en las potenciales situaciones de riesgo, influyendo en el funcionamiento general del individuo. Se destaca la influencia de variables predisponentes, como la historia familiar, el apoyo social, la personalidad, la historia de consumo, etc. Estas variables influirian en los procesos cognitivos, afectivos y de afrontamiento que se producirian en una situación específica de riesgo, serían variables de vulnerabilidad a la recaída y se les denomina en esta nueva reconceptualización factores distales. A los precipitantes que actuarían en una situación favoreciendo o evitando un consumo, se les denomina factores proximales. Estos precipitantes serian los procesos cognitivos, las estrategias de afrontamiento y los estados afectivos entre otros (Witkiewitz y Marlatt, 2004).

El curso longitudinal de la dependencia a la cocaína se caracteriza por la alternancia de períodos de abstinencia y recaídas. La oportunidad de disponer de factores predictivos del riesgo de recaída incentiva la realización de estudios e investigaciones que intentan establecer que influencia tienen éstos sobre el curso del tratamiento y sobre los resultados finales. El objetivo de este trabajo es el de revisar los últimos datos de la investigación relacionados con las variables y factores relacionados con un mayor riesgo de recaída en el consumo, en personas dependientes a la cocaína que realizan algún tipo de tratamiento para solucionar sus problemas de consumo. 


\section{Método}

Se realizó una búsqueda electrónica de trabajos que incluyeran el estudio de variables relacionadas con la recaída en el consumo de cocaína, en algunas de las bases de datos nacionales e internacionales más importantes en la actualidad: PubMed, ISI Web of Knowledge, The Cochrane Library, IME e ISOC. Para localizar los últimos datos de investigación se realizó una búsqueda de estudios publicados en el período comprendido entre Enero de 2000 a Julio de 2011. Se utilizó una combinación de los siguientes términos-descriptores de búsqueda: abuso, dependencia, adicción, trastorno por cocaina / recaída / abstinencia / variables predictivas / factores predictores / abandono del tratamiento / resultados de tratamiento. Una vez detectados los trabajos se realizó un cribado de los resúmenes de las publicaciones obtenidas con la estrategia de búsqueda. Se consideraron todos los trabajos que incluian para estudio a participantes que presentaran un diagnóstico de abuso o dependencia a cocaína, independientemente del patrón de consumo, sexo, edad o nacionalidad; y que estuvieran en tratamiento ambulatorio o residencial; aunque las muestras de estudio incluyeran a pacientes con un diagnóstico adicional de abuso o dependencia a otras sustancias. Debido a la gran cantidad de variables relacionadas con la recaída, los resultados se agruparon en relación al contexto actual de lo que se conoce sobre las recaídas, y se establecieron tres apartados diferentes: los correspondientes a la nueva reconceptualización de la naturaleza de las recaídas del clásico modelo de PR (prevención de recaídas), que distingue entre factores distales y proximales; y un nuevo apartado que engloba aquellos resultados que aparecieron relacionados con el tipo y los procesos de tratamiento. Dadas las dificultades y la falta de consenso sobre una definición clara de recaída también se consideraron los estudios en los que el abandono del tratamiento se consideró un fracaso, y se suponía por tanto una mayor probabilidad de recaída.

Hubo algunas restricciones relacionadas con el idioma de las publicaciones, ya que sólo se seleccionaron estudios publicados en inglés y castellano, debido a la dificultad de traducir trabajos en otros idiomas. También se excluyeron aquellos estudios que incluian informes de casos, estudios descriptivos, revisiones o aquellos que no especificaban el número de participantes o los criterios utilizados para realizar el diagnóstico. Del total de 113 artículos detectados, 54 cumplieron los criterios de selección y se consideraron para la revisión.

\section{Resultados}

\section{Factores Distales}

Los factores distales de recaída se suelen considerar como características del entorno y de la persona que abusa de sustancias, características que se asocian con una mayor vulnerabilidad a la recaída en algún momento en el futuro. Los estudios que investigan los factores distales utilizan diseños prospectivos, en el que las variables se evalúan en uno o más puntos de tiempo a través de auto-informes y entrevistas, así como con pruebas de laboratorio que proporcionan datos sobre factores de vulnerabilidad neurológicos y biológicos.

\section{Variables psicosociales}

Se considera que la falta de apoyo social puede ser uno de los factores que influyen de forma importante en la recaída durante y después del tratamiento (Garmendia, Alvarado, Montenegro y Pino, 2008). El apoyo social o nivel de apoyo emocional parece ser altamente predictivo de las tasas de abstinencia a largo plazo en varias conductas adictivas, incluyendo la dependencia a la cocaína (Dobkin, Civita, Paraherakis y Gill, 2001).

El contexto social en el que se desenvuelve la persona se considera crítico, un ambiente que favorezca el consumo multiplica las probabilidades de recaída (Hser, Evans, Huang, Brecht y Li, 2008). El tamaño de la red social relacionado con el consumo se relaciona de forma negativa con el abandono del uso de la sustancia (Buchanan y Latkin, 2008), mientras que la presión social aumenta las probabilidades de recaer (Ramo y Brown, 2008). El funcionamiento psicosocial deficiente puede estar relacionado con la recaída después del tratamiento, tanto en adolescentes (Anderson, Ramo, Schulte, Cummings y Brown, 2008) como en hombres y mujeres adultos (Sun, 2007). Un mejor funcionamiento social (p.ej. estar casado, buen nivel educativo, tener un trabajo) se asocia con mejores resultados en el tratamiento y menos recaídas (Simpson, Joe y Broome, 2002). Por el contrario, no estar casado y tener un bajo nivel educativo aumenta las probabilidades de recaer (Heinz, Wu, Witkiewitz, Eipstein y Preston, 2009). En estudios recientes (Fernandez-Montalvo y LopezGoñi, 2010; Santonja et al., 2010) se ha encontrado que el bajo nivel educativo y problemas familiares están directamente relacionados con menores tasas de retención en el tratamiento, y mayores probabilidades de recaída.

Varios predictores psicosociales de recaída entre los que se incluian la situación laboral habitual (peor pronóstico si se trabajaba a tiempo parcial) y el hecho de tener múltiples parejas sexuales se encontraron en un estudio de Greenwood et al., (2001). Se ha sugerido también que variables como los antecedentes de maltrato (Hyman, Paliwal y Sinha, 2007), y la presencia de estresores psicosociales (Tate, Brown, Glasner, Unrod y McQuaid, 2006) aumentan la probabilidad de recaida. Durante la abstinencia, la disponibilidad percibida de la sustancia en el contexto ambiental desempeña un papel importante en las respuestas de ansiedad y aumentan también las posibilidades de una recaída (MacKay et al., 2005; Tate et al., 2006)

Algunos estudios han documentado diferencias de género en las recaidas en la dependencia a cocaína, aunque los resultados son limitados. Por ejemplo, se señala que las mujeres parecer ser menos propensas que los hombres a experimentar recaídas (Gallop, Crits-Christoph, Ten-Have, Barber y Frank, 2007; Grella, Scott, Foss y Dennis, 2008), y que cuando se facilita el acceso a los servicios básicos y necesidades como vivienda y educación se reducen las probabilidades de 
recaída (VanDeMarck, 2007). En dos estudios realizados en población con problemática legal, las mujeres presentaron menores probabilidades de recaída durante el período de seguimiento (Johnson, Friedmann, Green, Harrington y Taxman, 2011; Pelissier, Camp, Gaes, Saylor y Rhodes, 2003).

\section{Comorbilidad Psiquiátrica}

La presencia de comorbilidad psiquiátrica (Messina, Farabee y Rawson, 2004; McMahon, 2008; Waldrop, Back, Verduin y Brady, 2007), incluyendo los trastornos de la personalidad (Fernández-Montalvo et al., 2004), también se ha relacionado con una mayor probabilidad de recaída. En un estudio reciente (Najt, Fusar y Brambilla, 2011), los peores resultados de tratamiento correspondieron a pacientes con trastorno por consumo de sustancias comórbido con trastorno depresivo mayor y pacientes con trastorno de estrés post-traumático. En cualquier caso, trabajos recientes señalan las dificultades para establecer qué tipo de variables predicen el mantenimiento en el tratamiento y la probabilidad de recaída en este grupo de pacientes (Brown, Bennet, Li y Bellack, 2011).

\section{Severidad Adictiva}

La severidad de la dependencia es otro de los factores relacionados con la recaída en el consumo de cocaína (Ahmadi et al., 2009). Se ha sugerido que la mayor severidad de problemas con el alcohol correlaciona con más probabilidad de recaídas en pacientes dependientes a la cocaína (Alterman et al., 2000), y también lo hace una mayor frecuencia de consumo de cocaína en los treinta días previos al inicio del tratamiento (Reiber, Ramirez, Parent y Rawson, 2002; Sofuoglu, Gonzalez, Poling y Kosten, 2003).

\section{Variables Biológicas y Neurobiológicas}

En los últimos años ha habido un considerable aumento de la investigación sobre la búsqueda de marcadores de pronóstico biológico de recaída en el consumo de cocaína (Gorelick et al., 2008; Kong y Xu, 2011; Mantsch et al., 2010). Algunos autores sugieren que la recaída puede estar relacionada con algunos de estos factores, incluyendo irregularidades en el sistema de la dopamina, problemas en la corteza prefrontal y regiones del cerebro relacionadas con el estrés y las señales condicionadas (Volkov et al., 2010). Sin embargo los resultados de los estudios biológicos son complejos y a veces contradictorios. El éxito de este tipo de trabajos ha sido hasta el momento limitado, pues en general se trata de pequeños estudios que no han sido replicados en muestras grandes o por distintos grupos de investigación (Gorelick et al., 2008).

\section{Factores Proximales}

En lugar de determinar los factores que indican una mayor vulnerabilidad a la recaída, los estudios de factores proximales buscan identificar las características y experiencias personales que se presume han provocado la aparición de un determinado episodio de recaída. Por lo tanto, estos estudios se centran en las cogniciones, las creencias, los estados de ánimo, las experiencias interpersonales, y otros factores presentes momentos antes 0 al inicio de una recaída. La mayor parte de la información sobre estos factores proviene de entrevistas e informes retrospectivos, aunque en los últimos años se están desarrollando con nuevas tecnologías otros métodos que mejoren la evaluación más clásica.

\section{Estados Afectivos}

Algunos autores han planteado que los afectos negativos son uno de los principales motivos para el consumo de drogas. (Baker, Piper, McCarthy, Mjeskie y Fiore, 2004). Varios estudios han documentado la existencia de un fuerte vínculo entre los estados afectivos negativos y las recaídas en el consumo (Fernandez, Jos, Lopez, Landa y Lorea, 2007; Stultz, Gallop, Lutz, Wrenn y Crist-Cristoph, 2011). La investigación ha mostrado vínculos particularmente fuertes entre la sintomatología depresiva y la recaída (Alterman, 2000; Witkiewitz y Bowen, 2010). El uso excesivo de sustancias podría estar motivado por la regulación afectiva, de manera que las sustancias proporcionaran refuerzo negativo cuando alivian los estados afectivos negativos (Tate, Brown, Unrod, y Ramo, 2004).

\section{Ansiedad-Craving}

Aunque la relación entre el deseo y el consumo ha mostrado resultados dispares en las recaídas a la cocaína, en parte debido a las dificultades para su medición (la mayoría de estudios utilizan una escala analógica visual o algunas preguntas directas), los estados de ansiedad relacionados con el deseo de consumo se ha señalado en diversos estudios como un fuerte predictor de recaida (Paliwall, Hymana y Sinha, 2008; Rohsenow, Martin, Eaton y Monti, 2007; Preston et al., 2009; Weiss et al., 2003). En estudios de laboratorio, la cantidad de ansiedad inducida por factores de estrés en sesiones experimentales, predice el tiempo hasta la reanudación del consumo de cocaína en la vida diaria (Sinha et al., 2006).

\section{Autoeficacia}

En general, la autoeficacia es un predictor de resultados en todos los tipos de conductas adictivas. La relación entre autoeficacia y resultados en el tratamiento de dependientes a cocaina ha sido examinada en varios estudios (Dolan, Martin y Rohsenow, 2008; Hser et al., 2006; Tate et al., 2008; Wong, Anthony, Mongeon, Badger, y Higgins, 2004). En dichos trabajos se concluye que aquellos pacientes que presentan niveles altos de autoeficacia parecen mostrar una mayor protección frente a la recaída. Los pacientes que manifiestan su intención de mantener una abstinencia total parecen tener menos probabilidades de recaída que los que no se plantean una abstinencia absoluta.

En el trabajo de Dolan et al. (2008), se encontró una asociación entre autoeficacia y un mayor deseo de dejar de consumir y menor necesidad de uso en situaciones de alto riesgo. La edad, los síntomas depresivos, el funcionamiento cognitivo, el consumo de sustancias recientes, y los éxitos 
del pasado en dejar el consumo también se correlacionaron con la autoeficacia. La autoeficacia también parece predecir el consumo de cocaína en usuarios de crack. Una mayor autoeficacia se ha relacionado con menor gravedad percibida del uso de sustancias y menor frecuencia de uso de alcohol (McKay et al., 2005).

\section{Factores relacionados con el tratamiento}

Además de las variables mencionadas anteriormente, otros factores relacionados con el tipo y el desarrollo de los tratamientos pueden influir en las recaidas y en los resultados de éstos. Por ejemplo, algunos estudios han mostrado que una menor intensidad en los servicios prestados y una menor asistencia a sesiones de seguimiento se relacionan con más probabilidades de recaer en el consumo (Grella, Hser y Hsieh, 2003; Hser, Joshi, Naglin y Fletcher 2004). Proporcionar servicios adicionales como acceso a vivienda y trabajo disminuye las recaídas (Milby et al., 2004). La misma relación se muestra cuando el uso de servicios psiquiátricos es menor (Ray, Weisner y Mertens, 2005), y cuando los pacientes presentan antecedentes de tratamientos previos (Dennis, Scott, Funk y Foss, 2005). En un estudio reciente, los pacientes que habian abandonado voluntariamente el tratamiento antes de su finalización, presentaron un riesgo superior de recaída que aquellos que finalizaban el tratamiento (Garmendia et al., 2008). Establecer sesiones de seguimiento una vez el tratamiento ha finalizado parece ser un factor protector de recaída (McKay, Merike, Mulvaney, Weiss and Koppenhauer, 2001), incluso si el seguimiento se hace a través de llamadas telefónicas (Godley, Coleman, Titus, Funk y Orndorff, 2010; McKay, Lynch, Shepard y Pettinati, 2005). Finalmente, la falta de motivación hacia el tratamiento y el escaso compromiso con la abstinencia, también aparecen el la literatura como variables predictoras del riesgo de recaída durante el tratamiento (Laudeck y Stanick, 2010).

\section{Discusión}

La recaída es un importante problema clínico y de investigación en las adicciones, para el que sin embargo no existe un consenso claro sobre su definición. La forma en que se mide la recaída puede producir diferencias sustanciales en la práctica clínica y en las conclusiones extraídas de los estudios empíricos (Maisto, Pollock, Cornelius, Lync y Martin, 2003). La falta de acuerdo sobre una definición operativa de la recaída, se ha debido tradicionalmente a las diferentes creencias sobre la etiología y el mantenimiento de los trastornos por uso de sustancias que subyacen a diferentes definiciones operacionales de la recaída (Marlatt y Gordon, 1985). Otra razón es la complejidad de obtener una definición operativa puede deberse a las múltiples dimensiones que deben ser consideradas en ella. Si la recaida se conceptualiza como el primer consumo de uso de sustancias, la relación con los resultados del tratamiento puede que tenga poco valor. Cuando la recaída se define de una forma más amplia, como el retorno a los niveles pretratamiento del uso de la sustancias, las recaídas y las medidas de resultados de tratamiento pueden estar mas estrechamente relacionadas (Warlitzer y Dearing, 2006).

En los últimos años ha habido un cambio en la investigación centrada principalmente en la evaluación retrospectiva de los factores que rodean la aparición de episodios de recaída, a un enfoque que también se dirige al estudio de los factores proximales. A pesar de la obvia importancia de los factores distales que aumentan la vulnerabilidad a la recaída de una manera general, la verdadera clave para entender porqué un episodio de recaída en particular se produce, probablemente se encuentra en las experiencias y los acontecimientos que surgen en las horas y los minutos previos al inicio de ese episodio. Por desgracia, la obtención de información precisa en ese periodo presenta enormes problemas metodológicos. Los tradicionales estudios prospectivos no están diseñados para recopilar datos inmediatamente antes de la recaída, y la precisión de la información en este período generada a través de estudios retrospectivos puede ser cuestionable. Para evitar la pérdida de información que supone el uso de cuestionarios o de datos retrospectivos, nuevas tecnologías están disponibles para evaluar en tiempo "casi real" las circunstancias que rodean la aparición de recaídas. Las técnicas en tiempo real incluyen registros diarios, teléfonos automatizados (IVR- Interactive Voice Response) (Collins, Kashdan, y Gollnisch, 2003); y computadoras de mano programadas (EMA- Ecological Momentary Assessment) (Shiffman, 2009). Estos enfoques pueden tener mejores perspectivas para la obtención de datos precisos en las horas previas a la recaída, pero hasta ahora sólo las recaídas en el tabaquismo han sido bien estudiadas con estas metodologías, ya que su uso presenta importantes dificultades prácticas para su aplicación (McKay, Franklin, Patapis y Lynch, 2006). En la actualidad se sigue estudiando la viabilidad de nuevos instrumentos; recientemente se ha desarrollado una escala para medir el riesgo de recaída a corto plazo en adultos (Kelly, Hoeppne, Urbanoski y Slaymaker, 2011).

La mayoría de los estudios sobre las recaídas se centran en un episodio específico, o en el consumo en un período de tiempo específico (por ejemplo seis a doce meses después de la línea de base). Sin embargo, muchos adictos a la cocaína sufren recaídas múltiples durante los episodios de tratamiento. En este sentido, hay muy poca información sobre la relación entre los factores distales y proximales que contribuyen a la aparición de episodios de recaída en el tiempo. No se sabe por tanto con certeza si los precipitantes de una primera recaída son los mismos que los de recaídas posteriores, o por el contrario si siguen un patrón diferente. Los resultados de algunos estudios (McKay et al., 2001) sugieren que los precipitantes claves de las recaídas puede cambiar con el tiempo.

En muchas recaídas hay un momento en el que la persona toma una decisión definitiva ya sea consciente o no, de seguir adelante y consumir la sustancia, pero ninguno de los métodos de evaluación es realmente capaz de capturar lo que está pasando en la mente de la persona en ese momento. La única manera de obtener información bastante detallada sobre los procesos de pensamiento, estados de ánimo, y las experiencias de recaída en los momentos previos es a través 
de entrevistas llevadas a cabo después de los hechos, aunque estos datos tienden a ser parciales (sesgos en las explicaciones e inexactitudes relacionadas con los recuerdos en el tiempo). No obstante este enfoque ofrece varias ventajas; es una manera fácil y relativamente barata de recoger datos sobre los precipitantes de recaída y los factores que influyen en su curso una vez ha comenzado y, los datos son a menudo extensos y ricos en detalles. Obviamente, este tipo de informes retrospectivos tienden a ser más precisos y completos cuando se obtienen poco después de iniciarse la recaída. Las dificultades para obtener este tipo de información tienen como consecuencia que los estudios sobre factores de vulnerabilidad (distales) son mucho más frecuentes que las investigaciones sobre factores proximales.

Por lo tanto, a pesar de algunos avances en las metodologías de evaluación disponibles para estudiar la recaída, todavía hay una razón convincente para no abandonar la práctica de pedir a la persona que describa la forma en que se produjo el episodio de recaída (McKay et al, 2006). Algunos estudios de seguimiento con pacientes dependientes a la cocaína realizados recientemente, apuntan a la complejidad de llevar a cabo este tipo de trabajos dadas las dificultades para contactar con los pacientes una vez el tratamiento ha finalizado, o con los pacientes que lo han abandonado (López et al., 2007; López et al., 2008). No obstante, el seguimiento de los pacientes y cualquier práctica dirigida a la monitorización rutinaria de los resultados puede ser beneficiosa, al permitir obtener información sobre los resultados de la intervención (Westbrock y Kirk, 2005; Wells, Saxon, Calsyn Jackon y Donovan, 2010).

Para finalizar podría decirse que en general ha habido desde un punto de vista teórico, un reconocimiento creciente de la compleja interacción y dinámica de los factores distales y proximales (Hufford, Witkiewitx, Shields, Kodya, y Caruso, 2003; Witkiewitz y Marlatt, 2004). No obstante y tal y cómo se ha mencionado anteriormente, por ser más compleja su evaluación, los estudios sobre factores proximales son más escasos. Existe un reconocimiento unánime en que la predicción y la comprensión de los procesos de recaída requiere un modelo multifactorial de tipo biopsicosocial, y existe un acuerdo general de que es el único enfoque conceptual que es razonable (Connors y Maisto, 2006).

\section{Perspectivas de futuro}

Hasta ahora una cantidad considerable de investigación sobre el abuso de sustancias ha examinado los factores que contribuyen a las recaídas, sin embargo es mucho más escasa la investigación que se ha realizado orientada a la identificación de los factores que protegen a los pacientes que no recaen. Se ha planteado la importancia de identificar los mecanismos psicosociales que subyacen a los procesos de remisión y recaída (apoyo social, reforzadores, habilidades de afrontamiento, etc), y determinar cómo estos y otros procesos comunes aumentan la probabilidad de remisión sostenida y la recuperación (Moos y Finney, 2011). Algunos autores ha llegado a plantear que los términos caída y recaída se basan en concepciones morales y religiosas de los problemas de drogas y deben ser reemplazados por términos moralmente más neutrales y precisos (Kelly y Westerhoff, 2010).

Los tratamientos no se agotan en el logro de la abstinencia, sino que también se dirigen a la mejora de la salud física y mental y a un mejor funcionamiento en los ámbitos laboral, familiar y educacional de la persona. El cuerpo de evidencia publicada en la actualidad está más centrado en la consecución de estos logros al finalizar el tratamiento, pero existe menor evidencia respecto a los factores que una vez lograda la abstinencia del consumo puedan favorecer o dificultar la permanencia de la misma. En un reciente estudio (Lopez-Quintero et al., 2011), se señala que el curso más probable de la dependencia de sustancias es la remisión, por lo que se plantea que la inmensa mayoría de individuos con dependencia a la cocaína superaran su dependencia en algún momento de su vida.

Estudios recientemente realizados, apuntan a la presencia de alteraciones en funciones como atención, memoria, habilidades psicomotoras y funciones ejecutivas en pacientes adictos a la cocaína (Garcia-Fernandez, Garcia-Rodriguez y Secades, 2011; Pedrero et al., 2011). La medición del funcionamiento cerebral durante la ejecución de diferentes tareas cognitivas puede proporcionar en el futuro un importante predictor de resultados de tratamiento en este tipo de pacientes. En cualquier caso aunque hace tiempo que se piensa que los déficits cognitivos y neuropsicológicos pueden afectar a las recaídas en el consumo de esta sustancia, la evidencia actual de una relación directa entre habilidades cognitivas y resultados del tratamiento está todavía por establecer.

Una cuestión en la que quizás sea importante profundizar es la importancia que el contexto ambiental pueda tener sobre el inicio o aumento del consumo en personas que no presentan problemas generados por el uso de cocaína. En este sentido se ha llegado a plantear en estudios recientes la hipótesis del "contagio social" entre consumidores y no consumidores (Sánchez-Hervás, Villanueva, Santonja, y Rubio, 2011; Santonja, Lombana, Rubio, Sánchez-Hervás y Villanueva, 2010).

\section{Limitaciones}

Dado que sólo se revisaron estudios publicados en inglés y castellano, es posible que algunos trabajos publicados en otros idiomas no se hayan considerado. Además, puesto que la revisión se centro en un período determinado (2000-2011), trabajos publicados con anterioridad a 2000 no se tuvieron en cuenta. Por otra parte, algunos estudios realizan seguimientos muy breves de los pacientes, por lo que es difícil establecer conclusiones definitivas sobre los resultados finales, dada la naturaleza recurrente de los procesos de recaída. En general, las diferentes formas de considerar o definir una recaída, dificultan la interpretación de algunos estudios, y aunque se dispone de mejores métodos de medición y análisis de datos, y ha aumentado la accesibilidad de éstos por parte de clínicos e investigadores, las dificultades para un análisis adecuado de las recaídas sigue estando presente. 


\section{Conclusiones}

Los problemas relacionados con el abuso de cocaína son difíciles de tratar, y muchas de las personas implicadas no consideran que el tratamiento sea necesario. Entre los que reciben tratamiento la recaída es algo común. En general, la recaída supone un importante desafío en el tratamiento de todos los trastornos de conducta, incluyendo los trastornos relacionados con el consumo de cocaína. Las personas involucradas en un proceso de cambio para abandonar la adicción a la cocaína se enfrentan a señales, impulsos y pensamientos automáticos que intentan modificar, por lo que las recaídas se convierten con frecuencia en algo complejo y en muchos casos impredecible. Las conceptualizaciones sobre los procesos de recaída han pasado de los modelos estáticos de factores de riesgo de recaída (Marlatt y Gordon, 1985) a modelos dinámicos que reconocen la naturaleza impredecible de la conducta de consumo de sustancias (Witkiewitz y Marlatt, 2004), donde pequeños cambios de retroalimentación en el sistema pueden provocar grandes cambios en relación al abuso de sustancias en un período relativamente corto de tiempo.

Podría decirse que una mejora de los conocimientos sobre los proceso de recaída se ha producido en los últimos años, y aunque la influencia de algunos factores sobre las recaídas en el consumo de cocaína parece clara, la investigación especifica es todavía escasa y no concluyente. Han sido varias las variables identificadas en la revisión relacionados con las recaidas en el consumo. Entre los factores distales se encontraron que las variables de tipo psicosocial tenian un importante valor predictivo, así como la comorbilidad psicopatológica y la severidad adictiva. Entre los factores proximales los estados emocionales negativos, ansiedad y autoeficacia, son las variables que con más frecuencia aparecen en los estudios publicados. Además, varios factores relacionados con el tratamiento como la intensidad y la oferta de los servicios prestados y las estrategias de seguimiento de casos, también se muestran como importantes factores predictivos. En conjunto, los resultados muestran la influencia de múltiples factores, aunque es difícil determinar el peso de cada factor por sí solo en el conjunto de la varianza total.

Las diferentes formas de delimitar e interpretar en que consiste una recaída, dificultan la realización de estudios cuyo objetivo específico sea la medición de las recaídas que se producen durante y después de los tratamientos, y posteriormente su interpretación. Es importante por tanto el considerar la recaída en el contexto más general del curso de la clínica, lo que podría facilitar la comprensión del proceso y su papel en el funcionamiento a largo plazo de los individuos, que en realidad es la principal preocupación de este campo de investigación.

\section{Conflicto de intereses}

Los autores manifiestan no tener ningún conflicto de intereses.
Tabla 1. Estudios utilizados en la revisión

\begin{tabular}{|c|c|c|c|c|}
\hline Autor & Año & Revista & Muestra & $\begin{array}{c}\text { Variables } \\
\text { Estudiadas }\end{array}$ \\
\hline Ahmadi et al & 2009 & Am J Addicions & $\begin{array}{c}\text { Dependientes } \\
\text { cocaina-alcohol } n=167\end{array}$ & $\begin{array}{c}\text { Severidad } \\
\text { adictiva }\end{array}$ \\
\hline Alterman & 2000 & Drug Alc Dependen & $\begin{array}{l}\text { Dependientes cocaina } \\
\qquad n=160\end{array}$ & $\begin{array}{l}\text { Estados } \\
\text { afectivos }\end{array}$ \\
\hline Anderson et al & 2008 & Substance Abuse & $\begin{array}{c}\text { Drogas ilegales y alcohol } \\
n=434 \text { (adolescentes) }\end{array}$ & $\begin{array}{l}\text { Funcionam. } \\
\text { social }\end{array}$ \\
\hline Brown & 2011 & Addictive Behaviors & $\begin{array}{l}\text { Drogas ilegales y } \\
\text { trastorno mental } n=175\end{array}$ & $\begin{array}{l}\text { Comorbilidad } \\
\text { psiquiátrica }\end{array}$ \\
\hline Buchanan y Latkin & 2008 & Drug Alc Dependen & $\begin{array}{l}\text { Abusadores cocaina } \\
\text { y heroina } n=629\end{array}$ & $\begin{array}{l}\text { Red } \\
\text { social }\end{array}$ \\
\hline Dennis et al & 2005 & JSubs Abus Treat & $\begin{array}{l}\text { Alcohol y drogas } \\
\text { ilegales } n=1271\end{array}$ & $\begin{array}{l}\text { Tratamientos } \\
\text { previos }\end{array}$ \\
\hline Dobkin et al & 2002 & Addiction & $\begin{array}{c}\text { Drogas ilegales y } \\
\text { alcohol } n=206\end{array}$ & $\begin{array}{l}\text { Apoyo } \\
\text { social }\end{array}$ \\
\hline Dolan et al & 2008 & Addictive Behaviors & $\begin{array}{l}\text { Dependientes } \\
\text { cocaina } n=163\end{array}$ & Autoeficacia \\
\hline Fernandez et al, & 2004 & In J Cli Health Psy & $\begin{array}{c}\text { Drogas ilegales y } \\
\text { alcohol } n=42\end{array}$ & $\begin{array}{l}\text { Comorbilidad } \\
\text { psiquiátrica }\end{array}$ \\
\hline Fernandez et al & 2007 & J. Addict. Diseases & $\begin{array}{c}\text { Drogas ilegales } \\
n=72\end{array}$ & $\begin{array}{l}\text { Estados } \\
\text { afectivos }\end{array}$ \\
\hline Fernandez et al & 2010 & Add Resear Theory & $\begin{array}{c}\text { Adictos cocaina } \\
n=102\end{array}$ & $\begin{array}{l}\text { Funcionam. } \\
\text { social }\end{array}$ \\
\hline Gallop et al & 2007 & J Cons Cli Psycholog & $\begin{array}{l}\text { Dependencia } \\
\text { cocaina } n=454\end{array}$ & Género \\
\hline Garmendia et al & 2008 & Rev. Med. Chile & $\begin{array}{l}\text { Dependencia drogas } \\
\text { ilegales y alcohol } n=306\end{array}$ & $\begin{array}{c}\text { Apoyo social } \\
\text { Cumplimiento del }\end{array}$ \\
\hline Godley et al & 2010 & J Sub Abus Treat & $\begin{array}{l}\text { Ábuso-Dependencia } \\
\text { alcohol y drogas } n=104\end{array}$ & $\begin{array}{c}\text { Sesiones } \\
\text { seguimiento }\end{array}$ \\
\hline Gorelick et al & 2008 & Psychopharmacology & $\begin{array}{l}\text { Abusadores de } \\
\text { cocaina } n=15\end{array}$ & $\begin{array}{l}\text { Marcadores } \\
\text { biológicos }\end{array}$ \\
\hline $\begin{array}{l}\text { Greenwood et al } \\
\text { Grella et al }\end{array}$ & $\begin{array}{l}2001 \\
2003\end{array}$ & $\begin{array}{l}\text { JSubs Abus Treat } \\
\text { JSubs Abus Treat }\end{array}$ & $\begin{array}{c}\text { Drogas ilegales } n=261 \\
\text { Abuso cocaina } \\
n=347\end{array}$ & $\begin{array}{l}\text { Situación laboral } \\
\text { Intensidad del } \\
\text { tratamiento }\end{array}$ \\
\hline $\begin{array}{l}\text { Grella et al } \\
\text { Heinz et al }\end{array}$ & $\begin{array}{l}2008 \\
2009\end{array}$ & $\begin{array}{l}\text { Evaluation Review } \\
\text { Addictive Behaviors }\end{array}$ & $\begin{array}{l}\text { Drogas ilegales } n=1202 \\
\text { Adictos cocaina } n=102\end{array}$ & $\begin{array}{c}\text { Género } \\
\text { Funcionamiento } \\
\text { social }\end{array}$ \\
\hline Hser et al & 2004 & Psychatr. Services & Drogas ilegales $n=1939$ & $\begin{array}{l}\text { Intensidad del } \\
\text { tratamiento }\end{array}$ \\
\hline Hser et al & 2006 & J Sbus Abus Treat & Adictos cocaina $n=266$ & Autoeficacia \\
\hline Hser et al & 2008 & Addictive Behaviors & Drogas ilegales $n=197$ & Contexto social \\
\hline Hyman et al & 2007 & Psych Add Behavio & $\begin{array}{l}\text { Dependientes cocaine } \\
\quad n=124\end{array}$ & $\begin{array}{l}\text { Antecedentes } \\
\text { de maltrato }\end{array}$ \\
\hline Johnson et al & 2011 & JSubs Abus. Treat & Drogas ileg. y alcohol $n=431$ & Género \\
\hline Laudet y Stanick & 2010 & J. Subs. Abus. Treat & Drogas ileg.-alcohol $n=250$ & Motivación \\
\hline McKay et & 2001 & Addiction & $\begin{array}{l}\text { Dependientes cocaina } \\
n=132\end{array}$ & $\begin{array}{l}\text { Sesiones de } \\
\text { seguimiento }\end{array}$ \\
\hline McKay et al & 2005 & J. Subs. Abus. Treat & $\begin{array}{l}\text { Drogas ilegales y } \\
\text { alcohol } n=132\end{array}$ & $\begin{array}{l}\text { Disponibilidad } \\
\text { Autoeficacia }\end{array}$ \\
\hline Mckay et al & 2005 & Arch Gen Psychiatry & $\begin{array}{l}\text { Dependientes alcohol } \\
\text { y cocaina } n=359\end{array}$ & $\begin{array}{l}\text { Sesiones de } \\
\text { seguimiento }\end{array}$ \\
\hline McMahon & 2008 & Am J Drug Alc Abus & $\begin{array}{l}\text { Dependencia cocaina } \\
\qquad n=304\end{array}$ & $\begin{array}{l}\text { Comorbilidad } \\
\text { psiquiátrica }\end{array}$ \\
\hline Messina et al & 2004 & J Cons Cli Psycholog & $\begin{array}{l}\text { Dependientes cocaina } \\
\text { en PMM } n=108\end{array}$ & $\begin{array}{l}\text { Comorbilidad } \\
\text { psiquiátrica }\end{array}$ \\
\hline Milby et al & 2004 & Psych Addic Behav & $\begin{array}{l}\text { Dependientes cocaina } \\
\text { sin techo } n=141\end{array}$ & $\begin{array}{l}\text { Servicios de } \\
\text { tratamiento }\end{array}$ \\
\hline Natj et al & 2011 & Psychiatr Research & $\begin{array}{l}\text { Revisión drogas y } \\
\text { comorbilidad }\end{array}$ & $\begin{array}{l}\text { Comorbilidad } \\
\text { psiquiátrica }\end{array}$ \\
\hline Paliwall et al & 2008 & Drug Alc Dependen & Depend. cocaina $n=123$ & Ansiedad-craving \\
\hline Pelisier et al & 2003 & I Subs Abus Treat & $\begin{array}{c}\text { Abuso alcohol y drogas } \\
n=2315\end{array}$ & Género \\
\hline Preston et al & 2009 & Psychopharmacolog & $\begin{array}{l}\text { Abuso cocaina en PMM } \\
n=112\end{array}$ & $\begin{array}{l}\text { Ansiedad- } \\
\text { craving }\end{array}$ \\
\hline Ramo et al & 2008 & PsycholAdd Behav & $\begin{array}{l}\text { Abuso de sust. y tr. psiq. } \\
n=160 \text { adult y } 188 \text { adol }\end{array}$ & $\begin{array}{l}\text { Presión } \\
\text { social }\end{array}$ \\
\hline Ray et al & 2005 & Psychiatric Services & $\begin{array}{l}\text { Drogas ilegales y } \\
\text { alcohol } n=604\end{array}$ & $\begin{array}{l}\text { Servicios de } \\
\text { tratamiento }\end{array}$ \\
\hline Reiber et al & 2002 & Drug Alc Dependen & $\begin{array}{l}\text { Dependendientes } \\
\text { cocaina } n=297\end{array}$ & $\begin{array}{l}\text { Severidad } \\
\text { adictiva }\end{array}$ \\
\hline Roshenow et al & 2007 & JStudies Alc Drugs & $\begin{array}{l}\text { Dependientes cocaina } \\
\qquad n=204\end{array}$ & $\begin{array}{l}\text { Ansiedad- } \\
\text { craving }\end{array}$ \\
\hline Santonja et al & 2010 & Add Dis The Treat & Depend. cocaina $n=70$ & Funcion. social \\
\hline Simpson et al & 2002 & Arch Gen Psychiatry & Dependientes cocaina $n=708$ & Funcion. social \\
\hline Sinha et al & 2006 & Arch Gen Psychiatry & Dependientes cocaina $n=54$ & $\begin{array}{l}\text { Ansiedad- } \\
\text { craving }\end{array}$ \\
\hline Sofuoglu et al & 2003 & Am J Drug Alc Abuse & $\begin{array}{l}\text { Depend. cocaina y opioides } \\
\qquad n=151\end{array}$ & $\begin{array}{l}\text { Severidad } \\
\text { adictiva }\end{array}$ \\
\hline Stulz et al & 2011 & Drug Alc Dependen & Dependencia cocaina $n=487$ & Estados afectivos \\
\hline & 2007 & & & \\
\hline Tat & 2004 & Addictiv & $=210$ & Estados afectivos \\
\hline & 2006 & Addic Reser Theory & Drogas ilegales-alcohol $n=102$ & $\begin{array}{l}\text { Stresores } \\
\text { psicosociales }\end{array}$ \\
\hline Tate et al & 2008 & Psychol Add Behav & Drogas ilegales $y$ alcohol $n=113$ & $\begin{array}{c}\text { Autoeficacia } \\
\text { Stress }\end{array}$ \\
\hline VanDeMarck & & Sub Abu $\operatorname{Tr} \operatorname{Pr} \& \operatorname{Pol}$ & Abuso drogas ilegales $n=325$ & \\
\hline Volkow et al & 2010 & Neuroimagen & Abuso cocaina $n=24$ & $\begin{array}{l}\text { Marcadores } \\
\text { biológicos }\end{array}$ \\
\hline Waldrop et al & 2007 & Addict & $\begin{array}{l}\text { Depend. cocaina-alcohol } \\
\qquad n=74\end{array}$ & $\begin{array}{l}\text { Comorbilidad } \\
\text { psiquiátrica }\end{array}$ \\
\hline $\begin{array}{l}\text { Weiss et al } \\
\text { Witkiewitz et al }\end{array}$ & $\begin{array}{l}2003 \\
2010\end{array}$ & $\begin{array}{l}\text { Am J Psychiatry } \\
\text { JCons Cli Psycholog }\end{array}$ & $\begin{array}{l}\text { Dependientes cocaina } n=449 \\
\text { Drogas ilegales } n=168\end{array}$ & $\begin{array}{l}\text { Ansiedad-craving } \\
\text { Estados afectivos }\end{array}$ \\
\hline Wong et al & 2004 & Exp Clin Psychophar & Dependientes cocaina $n=126$ & Autoeficacia \\
\hline
\end{tabular}




\section{Referencias}

Ahmadi. J., Kampman, K., Oslin. D., Pettinati, H., Dackis, C. y Sparkman, T. (2009). Predictors of treatment outcome in outpatient cocaine and alcohol dependence treatment. Amercian Journal Addictions, 18, 81-6. http://dx.doi.org/10.1080/10550490802545174

This article is not included in your organization's subscription. However, you may be able to access this article under your organization's agreement with Elsevier.

Alterman, A., Mckay, J., Mulvaney, F., Cnaan, A., Cacciola, J., Tourian, K. Rutherford, M. y Merikle, E. (2000). Baseline prediction of 7-month cocaine abstinence for cocaine dependence patients. Drug and Alcohol Dependence, 59, 215-21. http://dx.doi. org/10.1016/S0376-8716(99)00124-6

American Psychiatric Association (2000). Diagnostic and Statistical Manual of Mental Disorders DSM-IV-TR, 4th edition. Washington: APA.

Anderson, K., Ramo, D., Schulte, M. Cummings, K. y Brown, S. (2008). Impact of relapse predictors on psychosocial functioning of SUD youth one year after treatment. Substance Abuse, 29, 97-106. http://dx.doi.org/10.1080/08897070802093411

Baker, T., Piper, M., McCarthy, D., Majeskie, M. y Fiore, C. (2004). Addiction motivation reformulated: An affective processing model of negative reinforcement. Psychological Review, 11, 33-51. http://dx.doi.org/10.1037/0033-295X.111.1.33

Brown, C., Bennett, M., Li, L. y Bellack, A. (2011). Predictors of initiation and engagement in substance abuse treatment among individuals with co-occurring serious mental illness and substance use disorders. Addictive Behaviors, 36, 439-47. http://dx.doi. org/10.1016/j.addbeh.2010.12.001

Buchanan, B. y Latkin, C. (2008). Drug use in the social networks of heroin and cocaine users before and after drug cessation. Drug and Alcohol Dependence, 96, 286-289. http://dx.doi.org/10.1016/j. drugalcdep.2008.03.008

Collins, R., Kashdan, T. y Gollnisch, G. (2003). The feasibility of using cellular phones to collect ecological momentary assessment data: Application to alcohol consumption. Experimental and Clinical Psychopharmacology, 11, 73-78. http://dx.doi.org/10.1037//10641297.11.1.73

Connors, G. y Maisto, A. (2006). Relapse in addictive behaviors. Clinical Psychology Review, 26, 107-108. http://dx.doi.org/10.1016/j. cpr.2005.11.001

Cummings, C., Gordon, J. y Marlatt, G. (1980). Relapse: strategies of prevention and prediction. En Miller, W.R. (ed). The Addictive Behaviors: Treatment of alcoholism, Drug, Abuse, Smoking and Obesity. Oxford UK. Pergamon Press.

Dennis, M., Scott, C., Funk, R. y Foss, M. (2005). The duration and correlates of addiction and treatment careers. Journal of Substance Abuse Treatment, 28, S51-S62. http://dx.doi. org/10.1016/j.jsat.2004.10.013

Dobkin, P., Civita, M., Paraherakis, A. y Gill, K. (2002). The role of functional social support in treatment retention and outcomes among outpatient adult substance abusers. Addiction, 97, 347356. http://dx.doi.org/10.1046/j.1360-0443.2002.00083.x
Dolan, S., Martin, R. y Rohsenow, D. (2008). Self-efficacy for cocaine abstinence: pretreatment correlates and relationship to outcomes. Addictive Behaviors, 3, 675-688. http://dx.doi.org/10.1016/j. addbeh.2007.12.001

Dutra, L., Stathopoulou, G., Basden, S., Leyro, T., Powers, M. y Otto, M. (2008). A meta-analytic review of psychosocial interventions for substance use disorders. American Journal Psychiatry, 165, 179187

Fernández-Montalvo, J., López-Goñi, J.J., Landa, N., Illescas, C., Lorea, I. y Zarzuela, A. (2004). Trastornos de personalidad y abandonos terapéuticos en pacientes adictos: resultados en una comunidad terapéutica. International Journal of Clinical and Health Psychology, 4, 271-283.

Fernandez-Montalvo, J., Jos J., López-Goñi, J., Illescas, C., Landa, N. y Lorea, I. (2007). Relapse precipitants in addictions: results in a therapeutic community. Journal of Addictive Diseases, 26, 55-61.

Fernández-Montalvo, J. y López-Goñi, J. (2010). Comparison of completers and dropouts in psychological treatment for cocaine addiction. Addiction Research \& Theory, 18, 433-441. http://dx.doi. org/10.3109/16066350903324826

Gallop, J., Crits-Christoph, P., Ten Have, T., Barber, J. y Frank, A. (2007). Differential transitions between cocaine use and abstinence for men and women. Journal of Consulting and Clinical Psychology. 75, 95-103. http://dx.doi.org/10.1037/0022-006X.75.1.95

Garcia Fernandez, G., Garcia-Rodriguez, O. y Secades, R. (2011). Neuropsicología y adicción a drogas. Papeles del Psicólogo, 32, 159-165.

Garmendia, M., Alvarado, M., Montenegro, M. y Pino, P. (2008). Social support as a protective factor of recurrence alter drug addiction treatment. Revista Médica de Chile, 136, 169-178.

Garner, B. (2009). Research on the diffusion of evidence-based treatments within substance abuse treatment: A systematic review. Journal of Substance Abuse Treatment, 36, 376-399. http://dx.doi.org/10.1016/j.jsat.2008.08.004

Godley, D., Coleman-Cowger, H., Titus, C., Funk, R. y Orndorff, M. (2010). A randomized controlled trial of telephone continuing care. J. Subst Abuse Treat, 38, 74-82. http://dx.doi.org/10.1016/j. jsat.2009.07.006

Gorelick, D., Kyeong, Y., Bencherif, B., Boyd, S., Nelson, R., Copersino, M., Dannals, R. y Frost, J. (2008). Brain mu-opioid receptor binding: relationship to relapse to cocaine use after monitored abstinence. Psychopharmacology, 200, 475-486. http://dx.doi. org/10.1007/s00213-008-1225-5

Greenwood, G., Woods, W., Guydish, J. y Bein, E. (2001). Relapse outcomes in a randomized trial of residential and day drug abuse treatment. Journal of Substance Abuse Treatment, 20, 15-23. http://dx.doi.org/10.1016/S0740-5472(00)00147-1

Grella, C., Hser, Y. y Hsieh, S. (2003). Predictors of drug treatment re-entry following relapse to cocaine use in DATOS. Journal of Substance Abuse Treatment, 25, 145-154. http://dx.doi. org/10.1016/S0740-5472(03)00128-4

Grella, C., Scott, C., Foss, M. y Dennis, M. (2008). Gender similarities and differences in the treatment, relapse and 
recovery cycle. Evaluation Review, 32, 113-137. http://dx.doi. org/10.1177/0193841X07307318

Hair, J., Tatham, R., Anderson, R. y Black, W.C (2001). Multivariate data analysis. New York: Prentice Hall.

Heinz, A. J., Wu, J., Witkiewitz, K., Epstein, D. y Preston, K. (2009). Marriage and relationship closeness as predictors of cocaine and heroin use. Addictive Behaviors, 34, 258-263. http://dx.doi. org/10.1016/j.addbeh.2008.10.020

Hser, Y., Evans, E., Huang, D. y Anglin, D. (2004). Relationship between drug treatments services, retention, and outcomes. Psychiatric Services, 55, 767-774. http://dx.doi.org/10.1176/appi.ps.55.7.767

Hser, Y., Stark, M., Paredes, A., Huang D., Anglin, D. y Rawson, R. (2006). A 12-year follow-up of a treated cocaine-dependent sample. Journal of Substance Abuse Treatment, 30, 219-226. http://dx.doi. org/10.1016/j.jsat.2005.12.007

Hser, Y., Evans, E., Huang, D., Brecht, M. y Li, L. (2008). Comparing the dynamic course of heroin, cocaine, and methamphetamine use over 10 years. Addictive Behaviors, 33, 1581-1589. http://dx.doi. org/10.1016/j.addbeh.2008.07.024

Hufford, M., Witkiewitz, K., Shields, A., Kodya, S. y Caruso, J. (2003). Applying non-linear dynamics to the prediction of alcohol use disorder treatment outcomes. Journal of Abnormal Psychology, 112, 219- 227.

Hyman, S., Paliwal, P. y Sinha, S. (2007). Childhood maltreatment, perceived stress, and stress-related coping in recently abstinent cocaine dependent adults. Psychology of Addictive Behaviors, 21, 233-238. http://dx.doi.org/10.1037/0893-164X.21.2.233

Johnson, J., Friedmann, P., Harrington, M. y Taxman, F. (2011). Gender and treatment response in substance use treatment-mandated parolees. Journal of Substance Abuse Treatment, 40, 313-321. http://dx.doi.org/10.1016/j.addbeh.2011.05.013

Kelly, J., Hoeppne, B., Urbanoski, K. y Slaymaker, V. (2011). Predicting relapse among young adults: Psychometric validation of the advanced warning of relapse (AWARE) scale. Addictive Behaviors, $36,987-993$

Kelly, J. y Westerhoff, C. (2010). Does it matter how we refer to individuals with substance-related conditions? A randomized study of two commonly used terms. International Journal Drug Policy., 21, 202-201. http://dx.doi.org/10.1016/j. drugpo.2009.10.010

Kong $H_{1}$ y Xu, M. (2011). Exploring mechanisms underlying extinction of cue-elicited cocaine seeking. Curr Neuropharmacol, 9, 8-11. http://dx.doi.org/10.2174/157015911795017173

Laudet, A. y Stanick, V. (2010). Predictors of motivation for abstinence at the end of outpatient substance abuse treatment. Journal Substance Abuse Treatment, 38, 317-27. http://dx.doi. org/10.1016/j.jsat.2010.01.007

López, A., Becoña, E., Lloves, M., Moneo, A., Vieitez, I.,Cancelo, J.,... Díaz, E. (2007). Evolución del tratamiento y consumo en personas con dependencia de la cocaína, a los doce meses, en función de la psicopatología asociada y el funcionamiento cotidiano. Adicciones, 19, 251-266.
López, A., Becoña, E., Vieitez, I., Cancelo, J., Sobradelo, J., García, J.,... Lag, T. (2008). ¿Qué ocurre a los 24 meses del inicio del tratamiento en una muestra de personas con dependencia de la cocaina?. Adicciones, 20, 347-356.

Lopez-Quintero, C., Hasin, D., Pérez de Los Cobos, J., Pine,s A., Wang, S., Grant, B. et al. (2011). Probability and predictors of remission from life-time nicotine, alcohol, cannabis or cocaine dependence: results from the National Epidemiologic Survey on Alcohol and Related Conditions. Addiction, 106, 657-669. http://dx.doi. org/10.1111/j.1360-0443.2010.03194.x

Maisto, S., Pollock, N., Cornelius, J., Lyncn, K. y Martin, C. (2003). Alcohol relapse as a function of relapse definition in a clinical sample of adolescents. Addictive Behaviors, 28, 449-459. http:// dx.doi.org/10.1016/S0306-4603(01)00267-2

Maisto, S. y Connors, G. (2006). Relapse in the addictive behaviors: Integration and future directions. Clinical Psychology Review, 26, 229-231. http://dx.doi.org/10.1016/j.cpr.2005.11.009

Mantsch, J., Weyer, A., Vranjkovic, O., Beyer, C., Baker, A. y Caretta, H. (2010). Involvement of noradrenergic neurotransmission in the stress- but not cocaine-induced reinstatement of extinguished cocaine-induced conditioned place preference in mice: Role for beta-2 adrenergic receptors. Neuropsychopharmacology, 35, 2165-78. http://dx.doi.org/10.1038/npp.2010.86

Marlatt, A. Gordon, R. (1985). Relapse Prevention: maintenance strategies in the treatment of addictive behaviours. New York. Guilford Press.

McKay, J., Heirke, E., Mulvaney, F., Weiss, R. y Koppenhauer, J. (2001). Factors accounting for cocaine use two years following initiation of continuing care. Addiction, 96, 213-225. http://dx.doi. org/10.1046/j.1360-0443.2001.9622134.x

McKay, J., Foltz, C., Stephens, R., Leahy, P., Crowley, E. y Kissin, W. (2005). Predictors of alcohol and crack cocaine use outcomes over a 3-year follow-up in treatment seekers. Journal of Substance Abuse Treatment, 28, 73-82. http://dx.doi.org/10.1016/j. jsat.2004.10.010

McKay, J., Franklin, T., Patapis, N. y Lynch, K. (2006). Conceptual, methodological, and analytical issues in the study of relapse. Clinical Psychology Review, 26, 109-127. http://dx.doi. org/10.1016/j.cpr.2005.11.002

McKay, J., Lynch, K., Shepard, D. y Pettinati, H. (2005). The Effectiveness of Telephone-Based Continuing Care for Alcohol and Cocaine Dependence: 24-Month Outcomes. Arch Gen Psychiatry, 62, 199207. http://dx.doi.org/10.1001/archpsyc.62.2.199

McMahon, R. (2008). Substance abuse problems, psychiatric symptoms, and post treatment status in MCMI psychopathology subgroups of cocaine dependent. American Journal Drug Alcohol Abuse, 34, 195-202

Messina, N., Farabee, D. y Rawson, R. (2004). Treatment responsivity of cocaine-dependent patients with antisocial personality disorder to cognitive-behavioral and contingency management interventions. Journal of Consulting and Clinical Psychology, 71, 320-329. http://dx.doi.org/10.1037/0022-006X.71.2.320

Milby, J., Schumacher, J., Vuchinich, R., Wallace, D., Plant, M., Freedman, M. et al. (2004). Transitions During Effective Treatment 
for Cocaine-Abusing Homeless Persons: Establishing Abstinence, Lapse, and Relapse, and Reestablishing Abstinence. Psychology of Addictive Behaviors, 18, 250-256. http://dx.doi.org/10.1037/0893164X.18.3.250

Montoya, I. y Vocci, F. (2008). Novel medications to treat addictive disorders. Curr. Psychiatr. Rep, 10, 392-398. http://dx.doi. org/10.1007/s11920-008-0063-9

Moos, R. y Finney, J. (2011). Commentary on Lopez-Quintero et al. (2011): Remission and relapse - the Yin-Yang of addictive disorders. Addiction, 106, 670-671. http://dx.doi.org/10.1111/ j.1360-0443.2010.00003284.x

Najt, P., Fusar-Poli, P. y Brambilla, P. (2011). Co-ocurring mental and substance abuse disorders: A review on the potential predictors and clinical outcomes. Psychiatry Research, 186, 159-164.

Kelly, J. y Westerhoff, C. (2010). Does it matter how we refer to individuals with substance-related conditions? A randomized study of two commonly used terms. International Journal of Drug Policy, 21, 202-207. http://dx.doi.org/10.1016/j.drugpo.2009.10.010

Observatorio Europeo de las Drogas y las Toxicomanias (OEDT). (2010). 2010 Annual report on the state of the drugs problem in Europe. European Monitoring Centre for Drugs and Drugs Addiction. [EMCDDA]. Lisboa.

Observatorio Español sobre Drogas. (2009). Informe 2009. Situación y tendencias de los problemas de drogas en España. Ministerio de Sanidad y Politica Social. Secretaria General de Politica Social y Consumo. Delegación del Gobierno para el Plan Nacional sobre Drogas. Madrid.

Ochoa, E., Madoz-Gurpide, A. y Caballero, L. (2011). Cocaina. En: Manual de trastornos Adictivos. Bobes, J. Casas, M y Gutierrez, M. (eds). Adamed.

Oficina de las Naciones Unidas contra la Droga y el Delito (ONUDD). (2010). Informe Mundial sobre las Drogas. [World Drgu Report 2009]. United Nations Office on Drugs and Crime (UNODC). NY.

Pedrero, E., Rojo, G., Ruiz, J., Llanero, M., Garcia, G. y Puerta, C. (2011). Recuperación de la adicción: perspectiva neurológica y neuropsicológica. En: Neurociencia y Adicción. Pedrero, E (coord). Plan Nacional sobre Drogas. SET.

Pelissier, B., Camp, S., Gaes, G., Saylor, W., Rhodes, W. (2003). Gender differences in outcomes from prison-based residential treatment. Journal of Substance Abuse Treatment, 24, 149-160. http://dx.doi. org/10.1016/S0740-5472(02)00353-7

Paliwal, P., Hymana, S. y Sinha, R. (2008). Craving predicts time to cocaine relapse: Further validation of the Now and Brief versions of the cocaine craving questionnaire. Drug and Alcohol Dependence, 93, 252-259. http://dx.doi.org/10.1016/j.drugalcdep. 2007.10.002EscucharLeer fonéticamente

Polivy, J., y Herman, C. P. (2002). If at first you don't succeed: false hopes of self-change. American Psychologist, 57, 677-689. http:// dx.doi.org/10.1037//0003-066X.57.9.677

Preston, K., Vahabzadeh, M., Schmittner, J., Lin, J., Gorelick, D. y Epstein, D. (2009). Cocaine craving and use during daily life. Psychopharmacology, 207, 291-301. http://dx.doi.org/10.1007/ s00213-009-1655-8
Project MATCH Research Group. (1997). Matching alcoholism treatments to client heterogeneity: Project MATCH posttreatment drinking outcomes. Journal of Studies on Alcohol, 58, 7-29. http:// dx.doi.org/10.1037/0893-164X.22.3.372

Ramo, D. y Brown, S. (2008). Classes of Substance Abuse Relapse Situations: A Comparison of Adolescents and Adults. Psychology of Addictive Behaviors, 22, 372-379.

Ray, G., Weisner, C. y Mertens, J. (2005). Relationship between use of psychiatric services and five-year alcohol and drug treatment outcomes. Psychiatric Services, 56, 164-171. http://dx.doi. org/10.1176/appi.ps.56.2.164

Reiber, C., Ramirez, A., Parent, D. y Rawson, R. (2002). Predicting treatment success at multiple timepoints in diverse patients populations of cocaine-dependent individuals. Drug and Alcohol Dependence, 68, 35-48. http://dx.doi.org/10.1016/S03768716(02)00103-5

Rohsenow, D., Martin, R., Eaton, C. y Monti, P. (2007) Cocaine craving as a predictor of treatment attrition and outcomes after residential treatment for cocaine dependence. J Stud Alcohol Drugs, 68, 641-648.

Sánchez-Hervás, E., Villanueva, R., Santonja, F. y Rubio, M. (2011). Predicting cocaine consumption in Spain: A mathematical modelling approach. Drugs: Education, Prevention and Policy, 18, 108-115. http://dx.doi.org/10.3109/09687630903443299

Santonja, F; Sánchez-Hervás, E; Secades, R; Zacarés, F; GarcíaRodriguez, 0; Garcia-Fernandez, G. (2010). Pretreatment Characteristics as Predictors of Retention in Cocaine-dependent Outpatients. Addictive Disorders \& Their Treatment, 9, 93-98. http://dx.doi.org/10.1097/ADT.0b013e3181bff7ec

Santonja, F., Lombana, I., Rubio, M., Sánchez-Hervás, E. y Villanueva, J. (2010). A network model for the short-term prediction of the evolution of cocaine consumption in Spain. Mathematical and Computer Modelling, 52, 1023-1029. http://dx.doi.org/10.1016/j. mcm.2010.02.032

Shearer, J. (2007). Psychosocial approaches to psychostimulant dependence: a systematic review. Jounral Substance Abuse Treatmentt, 32, 41-52. http://dx.doi.org/10.1016/j.jsat.2006.06.012

Shiffman, S. (2009). Ecological momentary assessment (EMA) in studies of substance use. Psychological Assessment, 21, 486-497. http://dx.doi.org/10.1037/a0017074

Simpson, D., Joe, G. y Broome, K. (2002). A national 5-years followup of treatment outcomes for cocaine dependence. Archives of General Psychiatry, 59, 538-544. http://dx.doi.org/10.1001/ archpsyc.59.6.538

Sinha, R., Garcia, M., Paliwal, P., Kreek, J., Bruce J. y Rounsaville, M. (2006). Stress-induced cocaine craving and hypothalamicpituitary-adrenal responses are predictive of cocaine relapse outcomes. Archives General of Psychiatry, 63, 324-331. http:// dx.doi.org/10.1001/archpsyc.63.3.324

Sofuoglu, M., Gonzalez, G., Poling, J.y Kosten,T. (2003). Prediction of treatment outcome by baseline urine cocaine results and self-reported cocaine use for cocaine and opioid dependence. American Journal of Drug and Alcohol Abuse, 29, 713-727. http://dx.doi.org/10.1081/ADA-120026256 
Stulz, N., Gallop, R., Lutz, W., Wrenn, G. y Crits-Christoph, P. (2010). Examining differential effects of psychosocial treatments for cocaine dependence: An application of latent trajectory analyses. Drug and A/cohol Dependence, 106, 164-172. http:// dx.doi.org/10.1016/j.drugalcdep.2009.08.009

Stulz, N., Thaseb, M., Gallop, R. y Crits-Christoph, P. (2011). Psychosocial treatments for cocaine dependence: The role of depressive symptoms. Drug and Alcohol Dependence, 114, 41-48. http://dx.doi.org/10.1016/j.drugalcdep.2010.06.023

Sun, A. (2007). Relapse among substance-abusing women: components and processes. Substance Use \& Misuse, 42, 1-21.

Tate, S., Brown, S., Unrod, M. y Ramo, D. (2004). Context of relapse for substance-dependent adults with and without comorbid psychiatric disorders. Addictive Behaviors, 29, 1707-1724. http://dx.doi.org/10.1016/j.addbeh.2004.03.037

Tate, S., Brown, S., Glasner, S., Unrod, M. y McQuaid, J. (2006). Chronic life stress, acute stress events, and substance availability in relapse. Addiction Research and Theory, 14, 303-322. http:// dx.doi.org/10.1080/16066350500262817

Tate, S., Wu, J., McQuaid, J., Cummins, K., Shriver, C., Krenek, M. y Brown, S. (2008). Comorbidity of substance dependence and depression: role of life stress and self efficacy in sustaining abstinence. Psychology of Addictive Behaviors, 22, 47-57. http://dx.doi.org/10.1037/0893-164X.22.1.47

VanDeMark, N. (2007). Policy on reintegration of women with histories of substance abuse: A mixed methods study of predictors of relapse and facilitators of recovery. Substance Abuse Treatment, Prevention, and Policy, 2, 28. http://dx.doi. org/10.1186/1747-597X-2-28

Veach, L., Remley, T., Kippers, S. y Sorg, J. (2000). Retention predictors related to intensive outpatient programs for substance use disorders. American Journal of Drug and Alcohol Abuse, 26, 417-428. http://dx.doi.org/10.1081/ADA-100100253

Vocci, F. y Montoya, I. (2009). Psychological treatments for stimulant misuse, comparing and contrasting those for amphetamine dependence and those for cocaine dependence. Curr Opin Psychiatry, 22, 263-268. http://dx.doi.org/10.1097/ YCO.0b013e32832a3b44

Volkow, D., Fowler, J., Wang, G., Telang, F., Logan, J., Jayne, M., Ma, Y. et al. (2010). Cognitive control of drug craving inhibits brain reward regions in cocaine abusers. Neuroimagen, 49, 25362543.

Waldrop, A., Back, S., Verduin, M. y Brady, K. (2007). Triggers for cocaine and alcohol use in the presence and absence of posttraumatic disorder. Addictive Behaviors, 32, 634-639. http://dx.doi.org/10.1016/j.addbeh.2006.06.001

Walitzer, K, y Dearing, R. (2006). Gender differences in alcohol and substance use relapse. Clinical Psychology Review, 26:128148. http://dx.doi.org/10.1016/j.cpr.2005.11.003

Weiss, R., Griffin, M., Mazurick, C., Berkman, B., Gastfriend, D., Frank, A. et al. (2003). The relationship between cocaine craving, psychosocial treatment, and subsequent use. American Journal of Psychiatry, 160, 1320-1325. http://dx.doi.org/10.1176/appi. ajp.160.7.1320
Westbrock, D. y Kirk, J. (2005). The clinical effectiveness of cognitive behaviour therapy: outcome for a large sample of adults treated in routine practice. Behaviour Research and Therapy, 43, 1243-1261. http://dx.doi.org/10.1016/j.brat.2004.09.006

Witkiewitz, K. y Marlatt, G. A. (2004). Relapse preventions for alcohol and drug problems: That was Zen, this is Tao? American Psychologist, 59, 224-235. http://dx.doi.org/10.1037/0003066X.59.4.224

Witkiewitz, K. y Bowen, S. (2010). Depression, Craving, and Substance Use Following a Randomized Trial of Mindfulness-Based Relapse Prevention. Journal of Consulting and Clinical Psychology, 78, 362-374. http://dx.doi.org/10.1037/a0019172

Wong, C., Anthony, S., Sigmon, S., Mongeon, J., Badger, G. y Higgins, S. (2004). Examining interrelationships between abstinence and coping self-efficacy in cocaine-dependent outpatients. Experimental and Clinical Psychopharmacology, 12, 190-199. http://dx.doi.org/10.1037/1064-1297.12.3.190. 
